\title{
Entrenamiento virtual versus estándar de la reanimación cardiopulmonar neonatal y del lactante: revisión sistemática
}

\author{
Virtual versus standard training of cardiopulmonary resuscitation \\ for neonates and infants: a systematic review
}

Enrique Velásquez-Velásquez,* Juan Pablo Zapata-Ospina, *,‡ Diana Mora-Escallón, ${ }^{*, \ddagger}$ Daniel Felipe Patiño-Lugo*,ł

* Facultad de Medicina de la Universidad de Antioquia, ${ }^{\ddagger}$ Grupo Académico de Epidemiología Clínica (GRAEPIC), Medellín, Antioquia, Colombia.

\begin{abstract}
RESUMEN
Objetivo: Sintetizar la evidencia que compara los métodos presencial y virtual de enseñanza para la reanimación cardiopulmonar (RCP) neonatal y del lactante. Material y métodos: Revisión sistemática de ensayos clínicos aleatorizados (ECA), en los cuales se incluye personal de salud para la capacitación en RCP. Dos autores seleccionaron, sintetizaron y evaluaron la calidad de los estudios. No se realizó metaanálisis por la alta heterogeneidad entre estudios. Resultados: Se incluyeron cinco ECA que capacitaron a 315 personas: 160 virtual y 155 estándar. Los estudios son diferentes en cuanto a enseñanza virtual, tipo de participantes y evaluación de la efectividad de las intervenciones. Sin embargo, en cuanto a adquisición de conocimientos teóricos, parece no haber diferencias entre ambas técnicas educativas. En habilidades técnicas, la forma estándar produjo mejor desempeño global que usar video. Sobre habilidades no técnicas, la teleeducación generó niveles altos de satisfacción. Conclusiones: Son muy pocos ECA donde se ha comparado la modalidad virtual con la modalidad presencial para la enseñanza de la RCP de neonatos y lactantes. Por lo anterior, es necesario aumentar la investigación para determinar si la modalidad virtual es efectiva para mejorar conocimientos teóricos como habilidades para la RCP.
\end{abstract}

\begin{abstract}
Objective: To synthesize the evidence that compares face-to-face methods with virtual methods for teaching neonatal and infant cardiopulmonary resuscitation (CPR). Material and methods: Systematic review of randomized clinical trials (RCTs), in which health personnel are included for training in CPR. Two authors selected, synthesized and assessed the quality of the studies. Due to the high heterogeneity between studies, no metaanalysis was carried out. Results: Five RCTs that trained 315 people were included: 160 virtual and 155 standard. The studies are different in terms of virtual teaching, type of participants and evaluation of the effectiveness of the interventions. However, in terms of knowledge acquisition, there seems to be no difference between the two educational techniques. In technical skills, the standard form produced better overall performance than using video. On non-technical skills, tele-education generated high levels of satisfaction. Conclusions: There are very few $R C T s$ where the virtual modality has been compared with the face-to-face modality for teaching neonates and infants $C P R$. Therefore, it is necessary to increase research to determine if the virtual modality is effective for CPR, both in knowledge and skills.
\end{abstract}

Correspondencia: Enrique Velásquez-Velásquez, E-mail: enrique.velasquez@udea.edu.co

Citar como: Velásquez-Velásquez E, Zapata-Ospina JP, Mora-Escallón D, Patiño-Lugo DF. Entrenamiento virtual versus estándar de la reanimación cardiopulmonar neonatal y del lactante: revisión sistemática. Rev Mex Pediatr. 2021; 88(4): 133-142. https://dx.doi.org/10.35366/102777 
Número de registro PROSPERO: CRD42019116444.

Palabras clave: Reanimación cardiopulmonar, recién nacido, lactante, educación a distancia, revisión sistemática.

\section{INTRODUCCIÓN}

La mortalidad neonatal es un problema de salud pública a nivel mundial, pues representa $45 \%$ de las muertes infantiles y se calcula que tres de cuatro millones de muertes ocurren en la primera semana de vida, y un millón en el primer día. ${ }^{1,2}$ Hasta en $65 \%$ de los casos es posible prevenirla con diferentes medidas, en la que incluye a la asistencia efectiva en la transición de la vida intrauterina a la extrauterina.

La reanimación cardiopulmonar (RCP) neonatal es una de las intervenciones que facilita esta transición y reduce la mortalidad. ${ }^{3}$ Igualmente, los lactantes y escolares que presentan un paro cardiorrespiratorio (PCR) tienen alta mortalidad y su pronóstico puede ser incluso peor que en el adulto, independiente de la causa. ${ }^{4}$ No obstante, pareciera existir un mayor énfasis en el entrenamiento de RCP en adultos que en niños. ${ }^{5}$ Por ello es necesaria la capacitación del personal de salud, a fin de asegurar tanto la adaptación neonatal, como proporcionar una secuencia de RCP apropiada en niños y adolescentes.

Para la enseñanza de RCP en neonatos y lactantes, el método estándar en el mundo consiste en una forma tanto presencial como virtual (mixta), de acuerdo con la Academia Americana de Pediatría (AAP), que se basa en el International Liaison Committee on Resuscitation (ILCOR). ${ }^{6}$ Allí se detallan los procesos fisiopatológicos desencadenados durante un PCR en neonatos, en pediatría y en adultos, así como la evidencia respecto al método de RCP y las técnicas educativas para lograr la transferencia de conocimiento. En neonatos el método utilizado para RCP es el tradicional ABC (Air, Breath, Circulation), mientras que en pediatría y adultos es CAB (Circulation, Air, Breath). ${ }^{7}$

En general, el método instruccional utilizado en $\mathrm{RCP}$ en neonatos y pediatría inicia con la entrega de un texto guía al participante, en la cual se detalla cómo se debe preparar y realizar la reanimación. Dentro del material educativa, existe una aplicación virtual para familiarizarse con materiales y dispositivos. Al finalizar su lectura, el participante debe aprobar una prueba de conocimiento y, posteriormente asistir al entrenamiento presencial. En la sesión presencial se
PROSPERO Registration number: CRD42019116444.

Keywords: Cardiopulmonary resuscitation, newborn, infant, distance education, systematic review.

verifican conocimientos y habilidades en estaciones de aprendizaje diseñadas acorde a la edad objetivo, ya sea neonatal o pediátrica. Las sesiones incluyen la identificación y funcionamiento de equipos de ventilación, monitores, actividades en sala de partos, en la calle, cómo pedir ayuda, compresiones torácicas, accesos vasculares, identificación de arritmias, uso de desfibriladores, uso de fluidos y medicamentos. Además, se realizan actividades de simulación de casos clínicos para trabajo grupal, con un debriefing al final. La herramienta pedagógica que subyace es conocida como LSPPDM (de las siglas Learn, See, Practice, Prove, Do y Maintain) que es usada ampliamente en simulación para adquirir y mantener habilidades cognitivas, técnicas y de comportamiento. ${ }^{8}$

En países en vía de desarrollo, las sesiones presenciales para entrenamiento de RCP pueden tener limitaciones, como el un escaso número de instructores, requiere desplazamiento a los centros de entrenamiento y el número de participantes por instructor puede ser alto. Asimismo, los equipos de simulación pueden ser costosos y tornarse obsoletos ya que la tecnología está en constante evolución. Todo lo anterior impide disponer sesiones de capacitación frecuentes, ya que para mantener el conocimiento y habilidades se requiere actualizar capacitación cada tres a los seis meses; ${ }^{9}$ sin embargo, el ILCOR recomienda la recertificación cada dos años. ${ }^{10}$

Las dificultades señaladas abren la posibilidad de utilizar la capacitación virtual exclusiva para el aprendizaje de RCP, y no sólo como complemento al estándar. Sin embargo, su eficacia no se ha evaluado lo suficiente. ${ }^{3}$ En revisiones sistemáticas sobre la reanimación en adultos, se ha señalado que la formación virtual puede tener aceptabilidad por los participantes y mejorar las habilidades no técnicas ${ }^{11}$ y los conocimientos sobre $\mathrm{RCP},{ }^{12}$ aunque la evidencia aún es débil. El objetivo de esta revisión sistemática es sintetizar la evidencia proveniente de ensayos clínicos aleatorizados (ECA) para responder la pregunta: ¿cuál es la eficacia de la estrategia exclusivamente virtual en comparación con la enseñanza estándar para la capacitación de RCP de neonatos y lactantes al personal de salud, en términos de la adquisición de conocimientos y habilidades? 


\section{MATERIAL Y MÉTODOS}

El protocolo de investigación se inscribió en la base de datos PROSPERO (CRD42019116444). Mientras que para la escritura del presente artículo, se siguieron los pasos de Preferred Reporting Items for Systematic Reviews and Meta-Analyses (PRISMA).

\section{Criterios de elegibilidad}

Se incluyeron ECA que compararon la capacitación al personal de salud profesionales o en formación sobre RCP a neonatos y lactantes, mediante estrategias exclusivamente virtuales en contraste con la estrategia estándar.

\section{Fuentes de información}

La búsqueda se realizó en las bases de datos: PubMed, Cochrane Central Register of Controlled Trials (CENTRAL) y EMBASE, desde el 1 de enero del año 2000 a septiembre de 2019. Los ECA incluidos fueron solamente de publicaciones en idiomas inglés y español.

\section{Búsqueda}

Para la búsqueda en las bases de datos se usaron términos MeSH (Medical SubHeadings) y palabras en texto simple sobre: Cardiopulmonary Resuscitation, Infant, Newborn y los relacionados con educación. La estrategia de búsqueda completa puede verse en el Anexo 1.

\section{Selección de los estudios}

Los resultados de la búsqueda se exportaron a una plantilla de Microsoft Excel ${ }^{\circledR}$. Dos investigadores revisaron los registros, eliminaron los duplicados y los títulos no relacionados con el tema. Tanto los resúmenes como los artículos en extenso se evaluaron de forma independiente para identificar las publicaciones que cumplieran de los criterios de inclusión. Los desacuerdos se resolvieron por consenso. Se calculó el coeficiente kappa (к) para la concordancia inter-observador.

\section{Proceso de recolección de datos}

En una plantilla de Microsoft Excel ${ }^{\circledR}$ se recolectó la información de cada estudio: identificación, título, autor, año de publicación, características de la población, criterios de elegibilidad, número de participantes, características de la intervención, tipo de comparación y desenlaces.

\section{Evaluación de riesgos de sesgos}

Dos investigadores de forma independiente evaluaron el riesgo de sesgo de cada estudio y resolvieron desacuerdos por consenso. Se utilizó la herramienta de la colaboración de Cochrane. ${ }^{13}$ Para la concordancia inter-observador se calculó el coeficiente $\kappa$.

\section{Medidas de resumen y síntesis de resultados}

Se evaluaron tres desenlaces: 1) conocimientos teóricos alcanzados por los participantes, a través de exámenes de conocimientos; 2) habilidades técnicas, en las que se incluye el dominio en la realización de la RCP, con el uso de medidas correctivas de ventilación (como el acrónimo MRSOPA) en el caso de la ventilación con presión positiva acción o el desempeño global; y 3) habilidades no técnicas, que incluye la satisfacción de los participantes, manejo del estrés y conciencia situacional. No se realizó meta-análisis debido a la heterogeneidad, tanto de las intervenciones, del personal que fue capacitado, así como de la forma de evaluar el desenlace.

El sesgo de publicación no fue evaluado. Para la elaboración del gráfico de riesgo de sesgos se utilizó el software Review Manager 5.3. ${ }^{14}$

\section{RESULTADOS}

\section{Búsqueda y selección de los estudios}

La búsqueda arrojó 1,053 referencias y finalmente fueron seleccionadas cinco ECA (Figura 1). La concordancia entre evaluadores en cada una de las etapas para la selección fue alta $(\kappa=1.0)$.

\section{Características de los estudios}

En la Tabla 1 se observa que los cinco ECA seleccionados fueron diferentes. La intervención virtual utilizada fue de dos tipos: mediante video fue en tres estudios, ${ }^{15-17}$ y en dos ECA, mediante el "acompañamiento" por un experto. ${ }^{18,19}$ En total se incluyeron 315 participantes: 160 en el grupo virtual y 155 en el grupo estándar, pero el número por estudio varió de 42 a 88 . El tipo de participante también fue diferente entre los estudios: estudiantes de medicina, ${ }^{15}$ enfermeras ${ }^{16}$ residentes de pediatría ${ }^{18,19}$ y un terapeuta respiratorio. ${ }^{19}$ En tres estudios la capacitación fue para RCP neonatal, ${ }^{16,17,19}$ $\mathrm{y}$ en dos de lactante. ${ }^{15,18}$ 


\section{Descripción de las modalidades virtuales utilizadas}

Video para taller presencial: demostración por video de una secuencia de reanimación realizada por un instructor en un maniquí de un lactante, de 12 minutos de duración. ${ }^{15}$

Teleeducación: enseñanza de los contenidos teóricos y prácticos de la RCP, por medio de clases y demostraciones, en donde participantes e instructores interactúan a través de una pantalla de televisión a color. ${ }^{16}$

Video-feedback: consiste en la retroalimentación de la ejecución de la ventilación con presión positiva, a partir de la grabación en video. ${ }^{17}$
Asistencia remota con Google Glass (GG): durante la simulación de una PCR, el participante tiene videocomunicación por medio de unos lentes que transmiten audio y video, para comunicarse en tiempo real con el instructor (intesivista), quien guía el algoritmo de reanimación. ${ }^{18}$

Reanimación videoasistida (VAR): a través de la transmisión de video se permite la interacción, en tiempo real, durante la simulación de una PCR con un neonatólogo que hace recomendaciones o medidas correctivas sobre la ventilación y avances en flujograma. ${ }^{19}$

\section{Evaluación del riesgo de sesgos}

Los resultados para cada estudio están representados en la Figura 2. La concordancia entre evaluadores
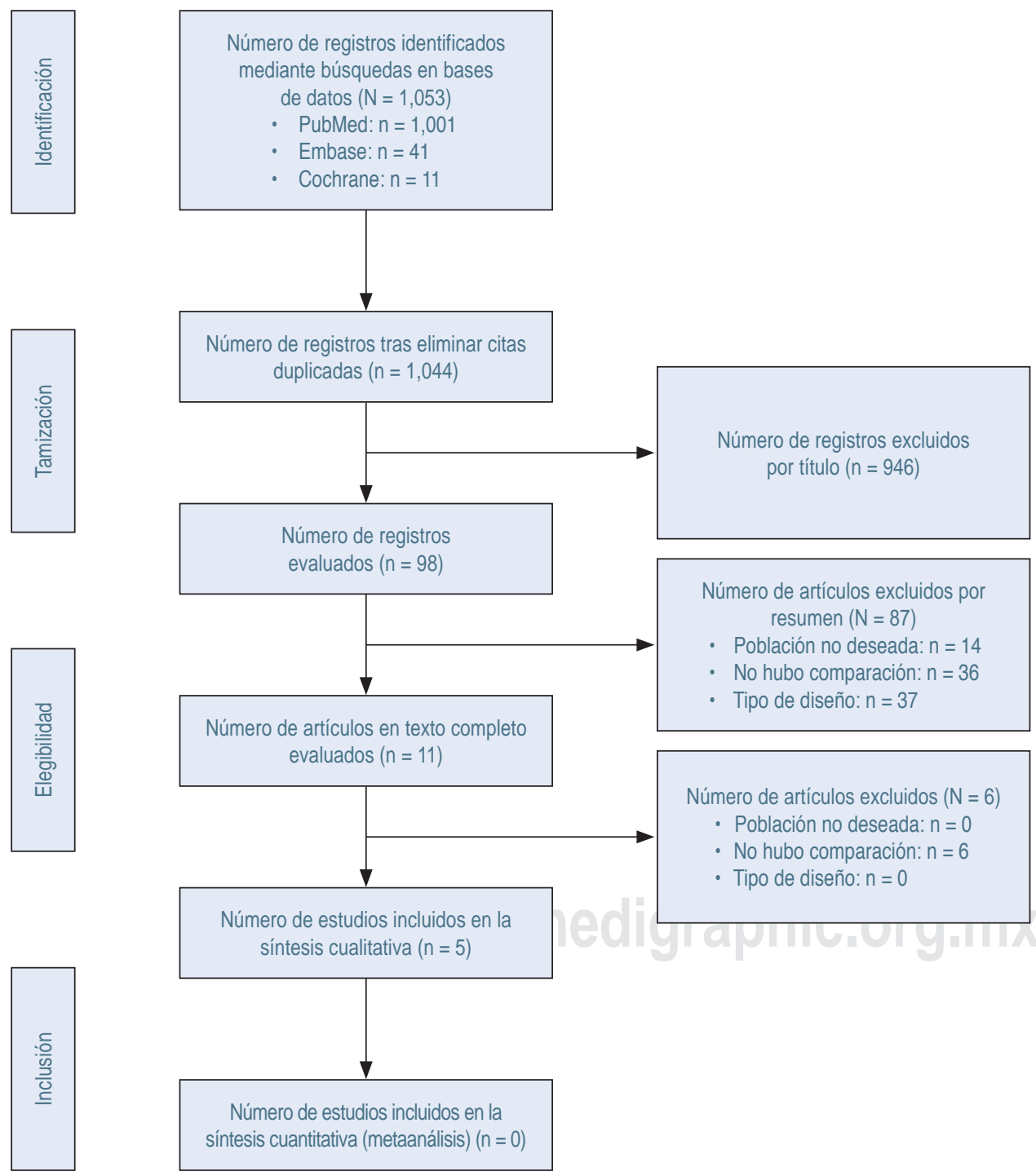

Figura 1:

Diagrama de flujo de la selección de artículos: se resume el proceso de búsqueda en las bases de datos $y$ selección final de los estudios. 
Tabla 1: Características de los ensayos clínicos que comparan la modalidad virtual y la estándar en la enseñanza de la reanimación cardiopulmonar (RCP) neonatal y pediátrica.

\begin{tabular}{|c|c|c|c|c|c|c|}
\hline \multirow[b]{2}{*}{$\begin{array}{l}\text { Artículo, } \\
\text { tipo de } \\
\text { reanimación }\end{array}$} & \multirow[b]{2}{*}{$\begin{array}{l}\text { Población } \\
\text { (n) }\end{array}$} & \multicolumn{2}{|c|}{ Intervención (n) } & \multicolumn{3}{|c|}{ Evaluación } \\
\hline & & Estándar & Virtual & Conocimientos & $\begin{array}{l}\text { Habilidades } \\
\text { técnicas }\end{array}$ & $\begin{array}{l}\text { Habilidades no } \\
\text { técnicas }\end{array}$ \\
\hline $\begin{array}{l}\text { Stephan, }{ }^{15} \\
\text { lactante en } \\
\text { RCP }\end{array}$ & $\begin{array}{c}\text { Estudiantes } \\
\text { de medicina } \\
\text { (88) }\end{array}$ & $\begin{array}{c}\text { Taller } \\
\text { presencial de } \\
12 \text { min (44) }\end{array}$ & $\begin{array}{l}\text { Teleeducación } \\
\text { por video de } 12 \\
\text { min del soporte } \\
\text { vital básico en } \\
\text { un lactante (44) }\end{array}$ & No realizada & $\begin{array}{l}\text { ECOE. Se evaluó } \\
\text { si estimula, llama, } \\
\text { posiciona, despeja } \\
\text { vía aérea y da } \\
\text { compresiones }\end{array}$ & $\begin{array}{l}\text { Satisfacción con } \\
\text { el método }\end{array}$ \\
\hline $\begin{array}{l}\text { Jain, }{ }^{16} \\
\text { neonato en } \\
\text { RCP }\end{array}$ & $\begin{array}{l}\text { Enfermeras } \\
\qquad(48)\end{array}$ & $\begin{array}{l}\text { Enseñanza } \\
\text { de RCP } \\
\text { con clase } \\
\text { tradicional } \\
\text { (22) }\end{array}$ & $\begin{array}{l}\text { Teleeducación: } \\
\text { evento } \\
\text { transmitido por } \\
\text { Internet (26) }\end{array}$ & $\begin{array}{c}\text { Test pre- y } \\
\text { postentrenamiento }\end{array}$ & $\begin{array}{l}\text { ECOE. Se evalúan } \\
\text { maniobras iniciales, } \\
\text { ventilación, sonda } \\
\text { orogástrica, } \\
\text { adrenalina y } \\
\text { aplicación del } \\
\text { algoritmo de RCP }\end{array}$ & $\begin{array}{l}\text { Satisfacción de } \\
\text { los alumnos, } \\
\text { participación. } \\
\text { Beneficio } \\
\text { personal, } \\
\text { capacidad } \\
\text { del docente, } \\
\text { satisfacción de } \\
\text { instructores }\end{array}$ \\
\hline $\begin{array}{l}\text { Amin, }{ }^{17} \\
\text { neonato en } \\
\text { RCP }\end{array}$ & $\begin{array}{l}\text { Proveedores } \\
\text { de RCP } \\
\text { certificados } \\
\text { (91) }\end{array}$ & $\begin{array}{l}\text { Feedback } \\
\text { revisando el } \\
\text { capítulo del libro } \\
\text { de RCP de la } \\
\text { AAP sin ver el } \\
\text { video (45) }\end{array}$ & $\begin{array}{l}\text { Video-feedback } \\
\text { con instructor, } \\
\text { con revisión } \\
\text { del video }(46)\end{array}$ & No realizada & $\begin{array}{l}\text { Ventilación efectiva } \\
\text { con máscara } \\
\text { mediante calificación } \\
\text { de } 13 \text { ítems al } \\
\text { terminar simulación } \\
\text { y luego de feedback }\end{array}$ & No realizada \\
\hline $\begin{array}{l}\text { Drummond, }{ }^{18} \\
\text { niño en } \mathrm{RCP}\end{array}$ & $\begin{array}{c}\text { Residentes } \\
\text { de pediatría } \\
\text { (42) }\end{array}$ & $\begin{array}{c}\text { RCP } \\
\text { estándar con } \\
\text { posibilidad de } \\
\text { llamar a un } \\
\text { especialista } \\
\text { (21) }\end{array}$ & $\begin{array}{l}\text { Gafas de } \\
\text { realidad virtual } \\
\text { aumentada } \\
\text { para } \\
\text { transmisión } \\
\text { con un } \\
\text { intensivista } \\
\text { como apoyo a } \\
\text { distancia } \\
\text { (21) }\end{array}$ & No realizada & $\begin{array}{l}\text { Tiempo sin } \\
\text { insuflación, sin } \\
\text { compresiones } \\
\text { torácicas. Despejar } \\
\text { vía aérea, retiro } \\
\text { de prong nasal, } \\
\text { eficiencia en VPP. } \\
\text { Técnica y frecuencia } \\
\text { del masaje cardiaco, } \\
\text { uso de adrenalina, } \\
\text { razón para interrumpir } \\
\text { insuflación o } \\
\text { compresión }\end{array}$ & No realizada \\
\hline $\begin{array}{l}\text { Fang, }^{19} \\
\text { neonato en } \\
\text { apnea }\end{array}$ & $\begin{array}{c}\text { Residentes de } \\
\text { pediatría (45) } \\
\text { y terapeuta } \\
\text { respiratorio } \\
\text { (1), } \\
\text { previamente } \\
\text { certificados en } \\
\text { RCP }\end{array}$ & $\begin{array}{l}\text { RCP estándar } \\
\text { (23) }\end{array}$ & $\begin{array}{c}\text { Asistencia } \\
\text { por video con } \\
\text { neonatólogo } \\
\text { (23) }\end{array}$ & No realizada & $\begin{array}{c}\text { Tiempo para } \\
\text { establecer } \\
\text { ventilación efectiva, } \\
\text { uso de MRSOPA }\end{array}$ & $\begin{array}{c}\text { Fallas } \\
\text { técnicas en la } \\
\text { implementación }\end{array}$ \\
\hline
\end{tabular}

MRSOPA = M (máscara), R (reposición), S (succión), O (open mouth), $\mathrm{P}$ (presión), A (airway alternative); VPP = ventilación con presión positiva; $A A P=$ Asociación Americana de Pediatría; ECOE = evaluación clínica objetivo estructurada. 
fue alta $(\kappa=0.81)$. Como se observa, con excepción del estudio de Jain y cols. ${ }^{16}$, el resto tuvo uno o más aspectos que pueden disminuir la calidad de los ECA. El estudio de Fang ${ }^{19}$ fue calificado con riesgo alto de sesgo en el cegamiento debido a que los calificadores estaban presentes en el momento de la evaluación inmediata de los participantes y conocían la intervención del neonatólogo de apoyo. En ese mismo estudio, en otros sesgos (inciso G) se calificó en rojo porque hubo desbalance inicial entre los participantes, pues el grupo de intervención tenía mayor experiencia.

\section{Conocimientos teóricos sobre RCP}

Sólo Jain y cols. ${ }^{16}$ realizaron evaluación de conocimientos antes y después del entrenamiento, con un test de 15 preguntas, con casos clínicos y preguntas de información específica. El grupo de tele-educación poseía un mayor conocimiento previo en comparación con el estándar (promedio inicial $8.3 \pm 1.7$ versus $6.6 \pm$ $1.4 ; \mathrm{p}=0.004)$. Después de la intervención, en los dos grupos hubo aumento en la puntuación, sin diferencia estadística entre grupos (promedio final 12.5 \pm 2.0 versus $12.0 \pm 1.7$, respectivamente; $p=0.06$ ).

\section{Habilidades técnicas en RCP}

Fueron evaluadas en los cinco estudios. En tres, se determinó un puntaje del desempeño durante la RCP
(Tabla 2). Stephan y cols. ${ }^{15}$ diseñaron una evaluación clínica objetiva estructurada (ECOE) de 12 preguntas, con un puntaje máximo de 24 puntos. Se consideró aprobada con 14 puntos y se realizó al terminar la intervención, entre dos y 10 semanas después. Los estudiantes de medicina del grupo estándar obtuvieron un mejor desempeño que los estudiantes del grupo de video, tanto en la evaluación inmediata (promedio de $19.0 \pm 0.4$ versus $17.3 \pm 0.5 ; \mathrm{p}=0.007$ ), como en la repetición ECOE semanas después.

Por su parte, Jain ${ }^{16}$ encontró que en el desempeño global en una ECOE ambos grupos de enfermeras mejoraron después de la intervención y no se encontraron diferencias significativas entre grupos; sin embargo, en un análisis multivariado, al ajustar por nivel basal de conocimientos teóricos, hubo significancia estadística de una diferencia pequeña (grupo estándar $15.6 \pm 2.5$ versus $16.0 \pm 2.8 ; \mathrm{p}<0.001$ ). En el estudio de Amin ${ }^{17}$ únicamente se evaluó la calidad de la ventilación, y se encontró que el desempeño con presión positiva fue estadísticamente mejor en el grupo de video-feedback al finalizar la intervención (Tabla 2).

Por su parte, Drummond ${ }^{18}$ evaluó habilidades técnicas de residentes de pediatría en la RCP del lactante; hubo dos evaluaciones, la primera ante un primer caso de PCR, y la segunda al implementar la GG en el grupo de intervención, con un nuevo caso clínico. Se encontró que con GG se alcanzó un mejor ritmo y posición en masaje cardiaco, así como superioridad en el retiro de
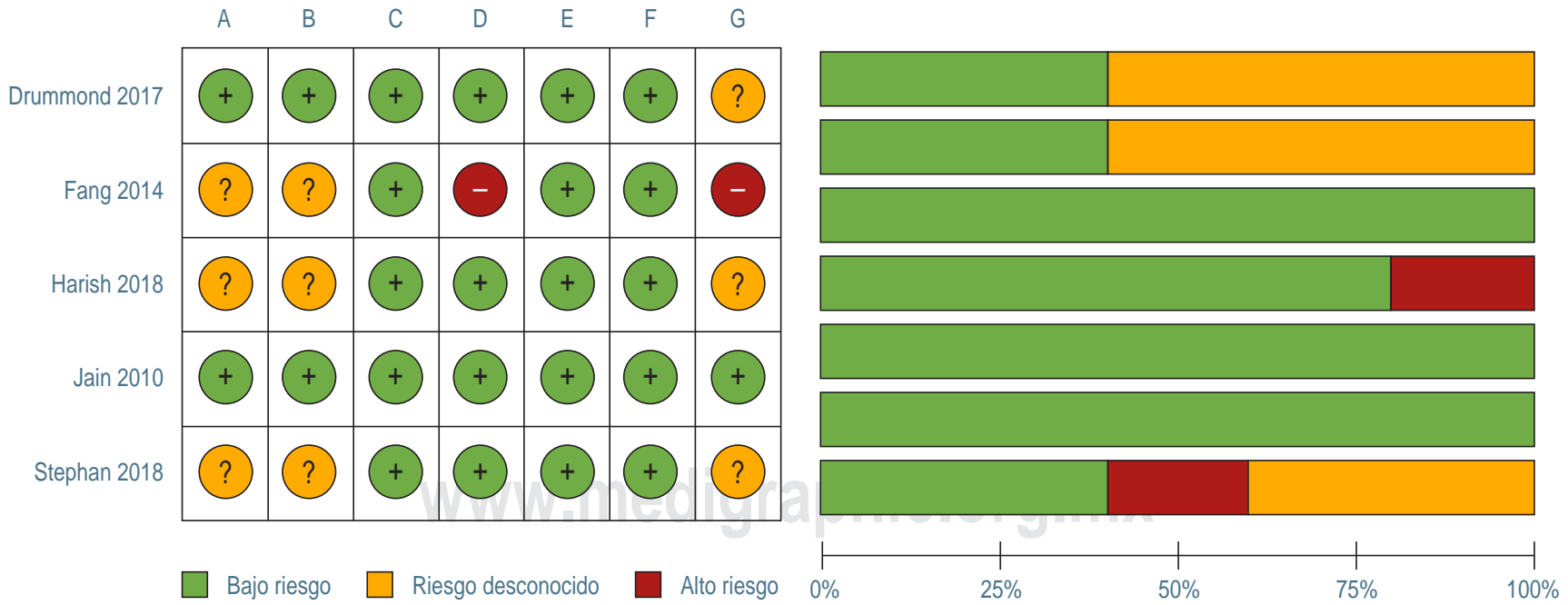

Figura 2: Calificación para cada estudio (izquierda) y resumen de la evaluación del riesgo de sesgos (derecha). Los ítems evaluados fueron: A. Generación de la secuencia de asignación aleatoria (sesgo de selección). B. Ocultamiento de la secuencia aleatoria. C. Cegamiento de participantes y personal (sesgo de desempeño). D. Cegamiento de evaluador de desenlace (sesgo de detección). E. Datos de desenlaces incompletos (sesgo de desgaste). F. Reporte selectivo de datos (sesgo de reporte). G. Otros sesgos. 
Tabla 2: Evaluación de habilidades técnicas en reanimación cardiopulmonar neonatal y pediátrica tras una intervención virtual en comparación con la estándar, en tres de los estudios incluidos.

\begin{tabular}{|c|c|c|c|c|c|c|}
\hline $\begin{array}{l}\text { Estudio e } \\
\text { intervención }\end{array}$ & $\begin{array}{c}\text { Puntaje } \\
\text { preintervención }\end{array}$ & p & $\begin{array}{c}\text { Puntaje } \\
\text { postintervención }\end{array}$ & $\mathbf{p}$ & $\begin{array}{l}\text { Puntaje en } \\
\text { seguimiento }\end{array}$ & $\mathbf{p}$ \\
\hline \multicolumn{7}{|c|}{ Stephan: $:^{15}$ desempeño en una ECOE } \\
\hline Virtual $(n=22)$ & NR & NR & $17.3 \pm 0.5$ & 0.008 & $18.6 \pm 0.5$ & 0.003 \\
\hline Estándar ( $\mathrm{n}=22)$ & NR & & $19.0 \pm 0.4$ & & $20.6 \pm 0.4$ & \\
\hline \multicolumn{7}{|c|}{ Jain: ${ }^{16}$ desempeño en una ECOE } \\
\hline Virtual $(n=26)$ & $11.7 \pm 3.0$ & 0.12 & $16.0 \pm 2.8$ & $0.55^{*}$ & NA & NA \\
\hline Estándar $(\mathrm{n}=22)$ & $10.3 \pm 2.9$ & & $15.6 \pm 2.5$ & & NA & \\
\hline \multicolumn{7}{|c|}{ Amin: ${ }^{17}$ lista de verificación sobre la ventilación con presión positiva } \\
\hline Virtual $(n=46)$ & 22.1 & NR & 25.1 & $<0.001$ & 24.7 & $<0.001$ \\
\hline Estándar $(n=45)$ & 20.1 & & 22.6 & & 21.6 & \\
\hline
\end{tabular}

Para cada estudio se presentan promedio \pm desviación estándar y los valores de $p$ correspondientes a la diferencia. Se anota el método usado para la evaluación de las habilidades. ECOE = evaluación clínica objetivo estructurada; NR = no reportado; NA = no aplica.

* Valor de p para la diferencia cruda; sin embargo, mediante análisis multivariado se ajustó por el nivel de conocimiento teórico basal, con valor $p<0.001$.

prongs nasales y mayor insuflación de manera eficiente. Para otras medidas del masaje cardiaco y uso de medicamentos no se encontraron diferencias (Tabla 3).

En el estudio de Fang ${ }^{19}$ se determinó el cumplimiento de cada paso del flujograma de la RCP neonatal, pero únicamente para la ventilación. En el grupo con VAR alcanzó la ventilación más rápido que el control (162 segundos versus 251 segundos; $\mathrm{p}<0.001$ ), además no se realizaron intubaciones innecesarias, a diferencia del control, que tuvo cumplimiento variable.

\section{Habilidades no técnicas en RCP}

Sólo el estudio de Jain y cols. ${ }^{16}$ comparó la satisfacción general sobre el uso de teleducación y la clase magistral, sin encontrar diferencias, En el estudio de Drummond ${ }^{18}$ no se realizó comparación en los problemas técnicos pues el control no usaba un aditamento comparable, pero se resalta que $29 \%$ de los participantes expresó que GG distraía de la RCP.

\section{DISCUSIÓN}

En esta revisión sistemática se encontró que la implementación de la tecnología en la enseñanza de la RCP pediátrica ha sido heterogénea. No obstante, para el conocimiento teórico, la estrategia virtual puede ser comparable con la forma presencial. En las habilidades técnicas, encontramos que el desempeño del personal que ya ha recibido entrenamiento en $\mathrm{RCP}$, la estrategia virtual da resultados similares, no así en personal no entrenado (estudiantes de medicina), en quienes no podría recomendarse. En cuanto a las habilidades no técnicas, si bien parece que la virtualidad es aceptada y percibida como satisfactoria, este desenlace no ha sido evaluado a profundidad.

Es necesario resaltar que solamente se identificaron cinco ensayos clínicos controlados que abordan la comparación de la educación virtual contra la estándar para la RCP. Es posible que el número tan limitado se deba a lo novedoso del tema, la resistencia que puede generar el reemplazo tecnológico en las actividades humanas, los paradigmas en pedagogía y la revolución digital misma. Sin embargo, estos estudios, en general, tienen buena calidad metodológica, pero existe heterogeneidad, lo cual impide determinar el efecto real de la estrategia virtual para la capacitación de RCP. Los participantes incluidos tenían diversos grados de experiencia; por ejemplo, enfermeras de hospitales de alta complejidad y residentes de pediatría podrían tener previamente mayores conocimientos y habilidades que los estudiantes de medicina. Los tipos de intervención también han sido variables, desde la enseñanza completa a distancia (teleeducación) y el video-debriefing hasta el contacto con especialistas en tiempo real por VAR y GG. Asimismo, la forma de evaluación de las habilidades técnicas fue diferente.

Solamente en un estudio se determinó que la educación virtual produce aumento en el conocimiento teórico de manera similar a la estrategia estándar. Es posible que la forma de transmitir contenidos no influya en el aprendizaje de conceptos y la memorización de algoritmos. Se ha sugerido que la educación virtual del 
personal de salud es equivalente a la presencial, con niveles de conocimientos comparables ${ }^{20}$ y de satisfacción. ${ }^{21}$ Tampoco la profundidad de los conocimientos no parece verse alterada por el hecho de impartirse de forma virtual. ${ }^{22}$ Por el contrario, la ventaja es que el material puede quedar almacenado y favorecer la actualización continua. ${ }^{23}$ De igual manera, con la tecnología se permite la conversación entre profesor y estudiante, lo cual seguramente afianza el conocimiento mediante la interacción entre ellos. ${ }^{24}$

En cuanto a las habilidades técnicas, es posible que con la educación virtual se mejore su desempeño, como la ECOE en personal que ya ha recibido entrenamiento, como se observó en enfermeras de hospitales de alta complejidad, del estudio de Jain y cols. ${ }^{16}$ En el estudio de Stephan ${ }^{15}$ se sugirió que el desempeño en habilidades técnicas fue mejor con la educación estándar; aunque la diferencia de puntajes fue pequeña. Esto podría indicar que en grupos con baja experiencia la enseñanza presencial es superior a la tele-educación. Sin embargo, vale la pena resaltar que, con el apoyo remoto en tiempo real de un especialista, el desempeño en la ventilación y el manejo de la vía aérea fue mejor que con el estándar. El contacto con el especialista por $\mathrm{GG}^{18}$ y VAR ${ }^{19}$ la ventilación efectiva y la realización de MRSOPA parecen ser mejores, hecho relevante pues, en la etapa neonatal, asegurar la ventilación es la medida más efectiva en reanimación y puede evitar la realización de procedimientos invasivo. ${ }^{25,26}$ Con estos resultados, se puede prever que, en los principiantes, el entrenamiento virtual podría mejorar el desarrollo de sus habilidades y aplicar exitosamente en una RCP real, en el futuro. ${ }^{27}$

Por otro lado, se debe mencionar que en un ECA se evaluó video-debriefing, que permitiría retroalimentación para una mejor realización de la secuencia de ventilación. Este proceso intencionado de aprendizaje es esencial en ambientes de simulación, ya que el participante aprende de los errores y puede mejorar el desempeño, a partir del retrato objetivo de lo ocurrido. ${ }^{27}$ También permite aumentar la confianza del participante. ${ }^{28}$

Es importante señalar que la aplicación de la tecnología a la enseñanza es un tema en evolución constante, por lo que en el futuro se podrá disponer de mayor número de investigaciones sobre las ventajas y desventajas de enseñanza a distancia. ${ }^{29-31}$ La ventaja de las formas virtuales radicaría en la posibilidad de usarlas de forma repetitiva, sin desplazamiento de los participantes o del instructor y facilitar la actualización. ${ }^{9}$ No obstante, esto exige investigación rigurosa para poder determinar si los nuevos métodos son eficaces, reproducibles y escalables, por lo que se requieren más estudios, ${ }^{32-34}$ dado el número tan limitado de ECA que se han realizado hasta ahora.

Dentro de las limitaciones de esta revisión se reconoce que la heterogeneidad de los estudios hace difícil llegar a una conclusión válida aplicable en todos los contextos. Igualmente, limitar la búsqueda a inglés y español, y sin haber localizado más artículos en fuentes de la literatura gris, podría generar incertidumbre sobre la posibilidad que exista más información.

Tabla 3: Evaluación de habilidades técnicas en la reanimación cardiopulmonar pediátrica con asistencia por lentes Google Glass (GG).

\begin{tabular}{|c|c|c|c|}
\hline Habilidad evaluada & $G G(N=21)$ & Control $(N=21)$ & $\mathrm{p}$ \\
\hline Retiro de prongs nasales, $\mathrm{n}(\%)$ & $20(95)$ & $10(48)$ & $<0.001$ \\
\hline Tiempo a la primera ventilación en segundos, m (RIQ) & $68(42-112)$ & $82(59-116)$ & 0.640 \\
\hline Fracción sin flujo, m (RIQ) & $49(39-60)$ & $53(41-76)$ & 0.280 \\
\hline Insuflación eficiente, $n(\%)$ & $20(95)$ & $14(67)$ & 0.040 \\
\hline Frecuencia cardiaca correcta 100-120 Ipm, n (\%) & $19(90)$ & $8(38)$ & $<0.001$ \\
\hline Posición correcta de manos, n (\%) & $21(100)$ & $15(71)$ & 0.020 \\
\hline Tiempo a la primera compresión en segundos, $m(R \mid Q)$ & $122(106-146)$ & $108(96-138)$ & 0.330 \\
\hline Fracción sin pulso, m (RIQ) & $68(60-77)$ & $73(63-94)$ & 0.280 \\
\hline Dosis correcta de adrenalina, $\mathrm{n}(\%)$ & $16(84)$ & $12(57)$ & 0.090 \\
\hline Tiempo para ordenar adrenalina, $m(R \mid Q)$ & $219(208-227)$ & $220(198-249)$ & 0.960 \\
\hline
\end{tabular}

Ipm = latidos por minuto; $m$ = mediana; RIQ = intervalo intercuartílico.

Los datos corresponden a los resultados presentados por Drummond. ${ }^{18}$ 


\section{CONCLUSIONES}

Los estudios que comparan la enseñanza de la RCP de neonatos y lactantes entre métodos virtuales exclusivos comparados con métodos estándar (presencial con y sin modalidad virtual) son muy limitados. Por lo anterior, son necesario más ECA para determinar la efectividad de la modalidad virtual, tanto en conocimientos teóricos como para la adquisición de habilidades técnicas.

\section{REFERENCIAS}

1. Gaffey MF, Das JK, Bhutta ZA. Millennium development goals 4 and 5: past and future progress. Semin Fetal Neonatal Med. 2015; 20: 285-292.

2. Lawn JE, Cousens S, Zupan J. 4 million neonatal deaths: When? Where? Why? Lancet. 2005; 365: 891-900.

3. Dempsey E, Pammi M, Ryan AC et al. Standardised formal resuscitation training programmes for reducing mortality and morbidity in newborn infants (Review). Cochrane Database Syst Rev. 2015.

4. Atkins DL, Everson-Stewart S, Sears GK et al. Epidemiology and outcomes from out-of-hospital cardiac arrest in children. Circulation. 2009; 119: 1484-1491.

5. Kumar VR, Bachman DT, Kiskaddon RT. Children and adults in cardiopulmonary arrest: are advanced life support guidelines followed in the prehospital setting? Ann Emerg Med. 1997; 29: 743-747.

6. American Heart Association. 2015 AHA Guidelines update for cardiopulmonary resuscitation (CPR) and emergency cardiovascular care (ECC) science. Circulation. 2015; 132: S519-S560.

7. Mancini ME, Diekema DS, Hoadley TA et al. Part 3: Ethical issues. Circulation. 2015; 132: S383-S396.

8. Ades A, Lee HC. Update on simulation for the Neonatal Resuscitation Program. Semin Perinatol. 2016; 40: 447-454.

9. Reisman J, Arlington L, Jensen L et al. Newborn resuscitation training in resource-limited settings: a systematic literature review. Pediatrics. 2016; 138: e20154490.

10. Ashish KC, Wrammert J, Nelin V et al. Evaluation of Helping Babies Breathe Quality Improvement Cycle (HBB-QIC) on retention of neonatal resuscitation skills six months after training in Nepal. BMC Pediatr. 2017; 17: 1-9.

11. Bracq MS, Michinov E, Jannin P. Virtual reality simulation in nontechnical skills training for healthcare professionals. Simul Healthc. 2019; 14: 188-194.

12. Lau $\mathrm{Y}$, Nyoe RSS, Wong $\mathrm{SN}$ et al. Effectiveness of digital resuscitation training in improving knowledge and skills: A systematic review and meta-analysis of randomised controlled trials. Resuscitation. 2018; 131: 14-23.

13. Higgins JP, Altman DG. Assessing risk of bias in included studies. In: Higgins JPT, Green S, eds. Cochrane handbook for systematic reviews of interventions. 2008 [Epub ahead of print 2008]. doi: 10.1002/9780470712184.ch8.

14. Review Manager (RevMan) [Software]. Version 5.4, The Cochrane Collaboration, 2020.
15. Stephan F, Groetschel H, Büscher AK, et al. Teaching paediatric basic life support in medical schools using peer teaching or video demonstration: A prospective randomised trial. J Paediatr Child Health. 2018; 54: 981-986.

16. Jain A, Agarwal R, Chawla D, et al. Tele-education vs classroom training of neonatal resuscitation: a randomized trial. J Perinatol. 2010; 30: 773-779.

17. Amin H, Singhal N, Beran T, et al. Use of simulation-based skill acquisition instruction (SSAI) for manual mask ventilation (MMV) improves the ability of neonatal resuscitation program (NRP) providers to deliver and retain effective MMV skills. Paediatr Child Health. 2018; 23: e48.

18. Drummond D, Arnaud C, Guedj R, et al. Google Glass for residents dealing with pediatric cardiopulmonary arrest: a randomized, controlled, simulation-based study. Pediatr Crit Care Med. 2017; 18: 120-127.

19. Fang JL, Carey WA, Lang TR, et al. Real-time video communication improves provider performance in a simulated neonatal resuscitation. Resuscitation. 2014; 85: 1518-1522.

20. Chipps J, Brysiewicz P, Mars M. A systematic review of the effectiveness of videoconference-based tele-education for medical and nursing education. Worldviews Evid Based Nurs. 2012; 9: 78-87.

21. Gonzalez-Espada WJ, Hall-Barrow J, Hall RW et al. Achieving success connecting academic and practicing clinicians through telemedicine. Pediatrics. 2009; 123: e476-e483.

22. Munyoka W. Evaluation impact of tele-education as new open distance learning delivery mode on learners in Botswana. Procedia - Soc Behav Sci. 2014; 116: 1248-1252.

23. Burke BL, Hall RW. Telemedicine: pediatric applications. Pediatrics. 2015; 136: e293-e308.

24. Holmberg B. Distance education theory again. Open Learning. 1997; 12: 31-39.

25. Davis PG, Dawson JA. New concepts in neonatal resuscitation. Curr Opin Pediatr. 2012; 24: 147-153.

26. Foglia EE, Te Pas AB. Effective ventilation: the most critical intervention for successful delivery room resuscitation. Semin Fetal Neonatal Med. 2018; 23: 340-346.

27. Arafeh JMR, Hansen SS, Nichols A. Debriefing in simulated-based learning. J Perinat Neonatal Nurs. 2010; 24: 302-309.

28. Fanning RM, Gaba DM. The role of debriefing in simulation-based learning. Simul Healthc J Soc Simul Healthc. 2007; 2: 115-125.

29. Silva AC, Bernardes A, Évora YD et al. Development of a virtual learning environment for cardiorespiratory arrest training. Rev ESC Enferm USP. 2016; 50: 990-997.

30. Totten A, Womack D, Eden K et al. Telehealth: Mapping the evidence for patient outcomes from systematic reviews. Rockville: Agency for Healthcare Research and Quality (AHRQ); 2016.

31. Dorsey ER, Topol EJ. State of telehealth. N Engl J Med. 2016; 375: 154-161.

32. Butler LC. The impact of telemedicine on teamwork and communication, workload, and clinical performance: a randomized controlled trial [Thesis]. USA: Yale University School of Medicine; 2017.

33. Fang JL, Campbell MS, Weaver AL et al. The impact of telemedicine on the quality of newborn resuscitation: a retrospective study. Resuscitation. 2018; 125: 48-55.

34. Colby CE, Fang JL, Carey WA. Remote video neonatal consultation: a system to improve neonatal quality, safety and efficiency. Resuscitation. 2014; 85: e29-e30. 
Anexo 1: Estrategia de búsqueda completa utilizada en cada base de datos.

\section{Base de datos}

PubMed

Cochrane Library

\section{Estrategia utilizada}

((Cardiopulmonary Resuscitation[MeSH] OR Resuscitation[tiab] OR CPR[tiab] OR NRP[tiab])

\section{AND}

(Infant, Newborn[MeSH] OR newborn[tiab] OR neonat[tiab] OR Infant[MeSH] OR infant[tiab]))

AND

(Education[MeSH] OR education[tiab] OR training[tiab] OR literacy[tiab] OR learn*[tiab])) OR (((Cardiopulmonary Resuscitation[MeSH] OR Resuscitation[tiab] OR CPR[tiab] OR NRP[tiab]) AND (Infant, Newborn[MeSH] OR newborn[tiab] OR neonat*[tiab] OR Infant[MeSH] OR infant[tiab])

AND

(Virtual Reality[MeSH] OR virtual[tiab] OR Telerehabilitation[MeSH] OR Telerehabilitation[tiab] OR e-learning[tiab] OR User-Computer Interface[MeSH] OR apps[tiab] OR phone[tiab] OR online[tiab] OR audiovisual[tiab] OR simulation[tiab] OR web[tiab] OR internet[tiab]))

"('resuscitation'/exp OR 'resuscitation') AND 'newborn'/exp

AND

('education'/exp OR 'virtual reality'/exp OR 'internet'/exp OR 'simulation'/exp)

AND

([cochrane review]/lim OR [systematic review]/lim OR [meta analysis]/lim OR [controlled clinical trial]/lim OR [randomized controlled trial]/lim)"

[Cardiopulmonary Resuscitation] explode all trees AND

[Infant, Newborn] explode all trees 